\title{
Variable structure pantograph mechanism with spring suspension system for comprehensive upper-limb haptic movement training
}

\author{
Joel C. Perry, PhD; ${ }^{1}$ Jakob Oblak; ${ }^{2}$ Je H. Jung, PhD; ${ }^{1}$ Imre Cikajlo, PhD ${ }^{2}$ Jan F. Veneman, PhD; ${ }^{1}$ Nika Goljar, \\ MD, PhD; ${ }^{2}$ Nataša Bizovičar, MD; ${ }^{2}$ Zlatko Matjačić, PhD; ${ }^{2}$ Thierry Keller, PhD $^{1^{*}}$ \\ ${ }^{1}$ Tecnalia Research \& Innovation, Health and Quality of Life Division, Donostia-San Sebastian, Spain; ${ }^{2}$ University \\ Rehabilitation Institute, Ljubljana, Slovenia
}

\begin{abstract}
Numerous haptic devices have been developed for upper-limb neurorehabilitation, but their widespread use has been largely impeded because of complexity and cost. Here, we describe a variable structure pantograph mechanism combined with a spring suspension system that produces a versatile rehabilitation robot, called Universal Haptic Pantograph, for movement training of the shoulder, elbow, and wrist. The variable structure is a 5-degree-of-freedom (DOF) mechanism composed of 7 joints, 11 joint axes, and 3 configurable joint locks that reduce the number of system DOFs to between 0 and 3. The resulting device has eight operational modes: Arm, Wrist, ISO (isometric) 1, ISO 2, Reach, Lift 1, Lift 2, and Steer. The combination of available work spaces (reachable areas) shows a high suitability for movement training of most upperlimb activities of daily living. The mechanism, driven by series elastic actuators, performs similarly in all operational modes, with a single control scheme and set of gains. Thus, a single device with minimal setup changes can be used to treat a variety of upper-limb impairments that commonly afflict veterans with stroke, traumatic brain injury, or other direct trauma to the arm. With appropriately selected design parameters, the developed multimode haptic device significantly reduces the costs of robotic hardware for full-arm rehabilitation while performing similarly to that of single-mode haptic devices. We conducted case studies with three patients with stroke who underwent clinical training using the developed mechanism in Arm, Wrist, and/or Reach operational modes. We assessed outcomes using Fugl-Meyer Motor Assessment and Wolf Motor Function Test scores showing that upper-limb ability improved significantly following training sessions.
\end{abstract}

Key words: arm therapy, haptic device, haptic training, pantograph mechanism, rehabilitation, rehabilitation robotics, series elastic actuation, stroke, stroke therapy, upper limb, wrist therapy.

\section{INTRODUCTION}

Rehabilitation robotics is a rapidly evolving field [1-3]. Numerous haptic devices have been developed for motor and neurological rehabilitation of upper limbs, and their effectiveness has been validated and presented in the last decade [4-22]. While improved effectiveness over conventional treatment has not been convincingly shown, in general, the leading advantages of robotic rehabilitation over traditional therapy are-

1. Task-oriented, consistent, and repetitive training.

2. Increased training duration and number of training sessions.

3. Broad range of training intensities.

4. Accurate objective measurement of patient progress based on numerical data (e.g., force and position).

5. Training motivation through games and virtual reality technology.

\footnotetext{
Abbreviations: $\mathrm{ADL}=$ activity of daily living, $\mathrm{DOF}=$ degree of freedom, FMA = Fugl-Meyer Motor Assessment, ISO = isometric, $\mathrm{ROM}=$ range of motion, $\mathrm{SEA}=$ series elastic actuator, UHD $=$ Universal Haptic Drive, UHP = Universal Haptic Pantograph, WMFT $=$ Wolf Motor Function Test.

*Address all correspondence to Thierry Keller, PhD; Tecnalia Research \& Innovation, Mikeletegi Pasealekua 1, E-20009 Donostia-San Sebastian, Spain; +34-667-119652; fax: +34-946-460900. Email: thierry.keller@tecnalia.com DOI:10.1682/JRRD.2010.03.0043
} 
A survey of literature published to date reveals that the kinematic structure of robots for upper-limb motor rehabilitation is commonly either (1) serial linkage mechanisms in which the end effector of the robot contacts the user's hand, suitable for either arm-reach movement [411] or wrist-movement [12-16] training, or (2) exoskeleton-based mechanisms that provide haptic interaction for both the arm and wrist simultaneously [17-20]. Serial mechanism devices typically have between 1 and 3 degrees of freedom (DOFs), with the exception of those that have additional DOFs for wrist movement [21-22], while exoskeleton mechanisms typically have 7 DOFs. In general, a high number of DOFs in an exoskeleton mechanism trades affordability for functional versatility, impeding widespread use. On the other hand, clinical use of low-DOF serial mechanisms requires the use of two or more devices for complete arm-movement training. This approach increases the overall cost of the rehabilitation system by requiring greater financial and spatial resources, as well as additional therapists' time to familiarize themselves with multiple machines. As an alternative, a pantograph kinematic structure may be considered.

A pantograph kinematic structure is a four-bar parallel linkage through which movements are induced at one point and a scaled copy is reproduced at another point. Historically, pantographs have been used for copying and resizing images and text. In this case, the pantograph structure is used to transmit scaled forces from two actuators to an arm or hand for rehabilitation.

Numerous clinicians have expressed the desire to use robotic rehabilitation technologies if priced affordably, but many institutions lack the finances to purchase existing arm-rehabilitation equipment that can cost more than $\$ 50,000$ a unit. As a result, a strong need exists for the development of haptic rehabilitation robots that use fewer DOFs, thus reducing the costs of hardware, while still offering the possibility of training arm-reach as well as wrist-movement tasks. This need is believed to be satisfied through an innovative rehabilitation robot designed with a redundant set of joints and joint locks in a pantograph kinematic structure, allowing multiple system configurations to be controlled by the same set of actuators.

So far, few rehabilitation robots have implemented a pantograph structure in their design, perhaps because of their inherently limited workspace. On the other hand, pantograph mechanisms are composed of numerous joints connected through parallel linkages, in which the number of DOFs of the overall system is reduced by the connections between the joints. As a result, system DOFs are always fewer than the sum of individual joint axes, which means that configurable joint connections could leverage the higher number of joints contained within the overall system. Similarly, this concept can be extended to a device with higher DOFs. If the mechanical joints of a pantograph mechanism, for example, are designed so that they can be easily locked or unlocked (thus limiting DOFs of a particular joint), a mechanism may be conceived that could enable movement training of various aspects of the upper limb (e.g., arm reaching, wrist orienting, etc.) by enabling the therapist to select appropriate modes of individual joints of a mechanism while maintaining the same number of DOFs of the whole system. An additional benefit from such a design is that with only 2 DOFs of actuation (a costly part of robotic hardware), the two actuators could be used for all required movement training. Therefore, a pantograph mechanism with an appropriate system of joints and locks can significantly improve the cost-effectiveness of robotic devices for full-arm rehabilitation.

In this article, we first present an essential background related to the development of an arm-rehabilitation device called the Universal Haptic Pantograph (UHP), particularly its developmental predecessor, an existing haptic rehabilitation robot for upper-limb training called the Universal Haptic Drive (UHD). Following the background information, we describe the UHP design, along with the kinematics of the mechanism in its various configurations. Finally, we present outcome measures from three clinical case studies by means of Fugl-Meyer Motor Assessment (FMA) and Wolf Motor Function Test (WMFT) scores.

\section{BACKGROUND}

In robot-mediated upper-limb therapy, the range of motion (ROM) of the arm controlled by a rehabilitation robot is one of the crucial metrics used to measure efficacy of movement training. Hence, rehabilitation robots are generally designed based on the ROM requirements to perform activities of daily living (ADLs), for which the average and peak values of angles and torques seen in the shoulder, elbow, and wrist during these activities have been previously investigated. Studies acknowledge that different arm tasks involve varying amounts of proximal and distal arm joints, but whether optimal arm rehabilitation should include simultaneous training for the shoulder, 
elbow, and wrist is still debated. However, growing evidence suggests that separating wrist from shoulder and elbow training is justified and, furthermore, that robotics needs only to accommodate a subset of the arm workspace for effective training of many ADL tasks.

A recent research result from a study on the kinematics and dynamics of the human arm during ADLs (24 arm activities) in a free and unconstrained environment shows that although some complex and partially constrained tasks, such as brushing the teeth or opening a cupboard, require moving shoulder, elbow, and wrist joints simultaneously, many tasks rely on the serial combination of shoulder and elbow movement and wrist movement [23]. The shoulder and elbow are commonly first used for positioning an object relative to the environment or positioning the hand relative to an object. Then, with the hand positioned in a desired location relative to the environment, the wrist is used for orienting the hand and/or other objects with respect to one another. This observation supports separating shoulder and elbow training from wrist training for most tasks. In addition, the feasible training workspace derived from pick-and-place experiments in nondisabled populations has shown that a human moves his or her arm toward a given target position in a way that requires only 2 DOFs, and the amplitude of these movements is limited to $20 \mathrm{~cm}$ from the body [24].

Other relevant evidence has demonstrated that supporting the arm against gravity enables subjects with stroke to increase movement ranges compared with unsupported motion [25]. Furthermore, studies have shown that training arm-reach movements under progressive abduction loading of the shoulder (requiring the subject to maintain a load against gravity) enhance the reaching ROM [26]. In particular, the largest ROM improvements were recorded for abduction-loading levels between 100 and 175 percent of limb weight. In other words, to facilitate movement in subjects with impairment, a device needs to offer variable gravitational support with the capacity to support partial limb weight as well as to impose loading levels above that induced by the weight of the arm itself. In this way, the loading on the arm can be tailored to the ability of patients and the phase of their recovery.

The results just described strongly support the development of a simpler robotic design with a higher probability for cost-effective rehabilitation. Reflecting these results, the UHD (a low-cost configurable system) was previously developed (Figure 1(a)) and tested for train- ing both the upper arm and wrist separately with the same 2 controlled DOFs for each configuration [27]. The system used a configurable joint lock to allow the 2 actuated DOFs to be used selectively in one of two modes: (1) Arm mode, for quasiplanar arm positioning tasks, or (2) Wrist mode, for exercising wrist pronosupination, flexion-extension, or radial-ulnar deviation. In Wrist mode, a bar with a forearm orthosis attaches to the base to support the forearm against gravity, as shown in Figure 1(a). In the Arm mode, this bar is removed. Pre- and posttreatment results from a one-subject pilot study (rightsided hemiparesis, 10-year poststroke) significantly improved wrist radial deviation, supination, and extension, as well as in two of eight directional movements for arm positioning. Training sessions were administered on 6 consecutive days, and each training session lasted approximately 45 minutes.

After successfully developing and testing this first system, we found a number of potential design revisions that would increase the number of functional training modes available, facilitate easier switching between modes, improve the means of providing gravity compensation to the arm, and better facilitate shoulder abduction loading. As a result, the first UHD system was redesigned, and an innovative pantograph mechanism and spring suspension system were implemented. Throughout the remainder of this article, the revised system will be referred to as the UHP.

\section{METHODS}

The developed UHP system (Figure 1(b)) is a thirdgeneration prototype, built from previously published work on the UHD, which was described in detail by Oblak et al. in 2010 [27]. While the UHP uses the same series elastic actuators (SEAs) as used in the UHD (SEA also described by Oblak et al. [27]), the modification can be found in a pantograph mechanism and system of configurable joint locks that can more efficiently transition between five distinct modes of operation with minimal effort by the therapist.

\section{Device Description}

The overall design and user interface of the UHP are presented in Figure 2. The UHP uses a pantograph mechanism composed of two vertical bars (Actuated and Parallel Bars) and a horizontal bar (Transverse Assembly) to which the user's hand and forearm are attached. The 
(a)

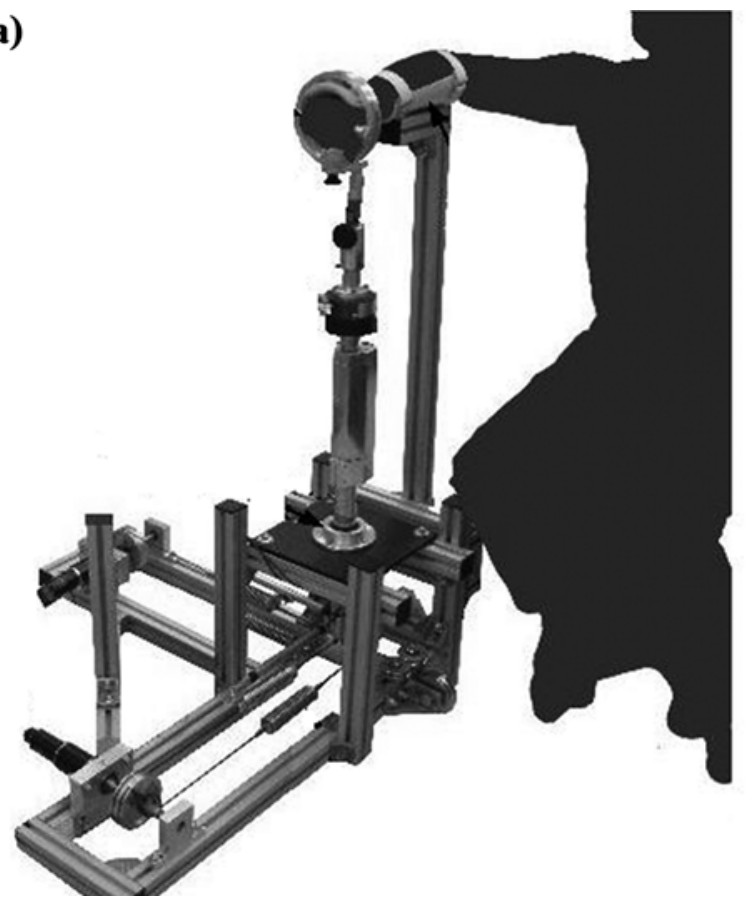

(b)

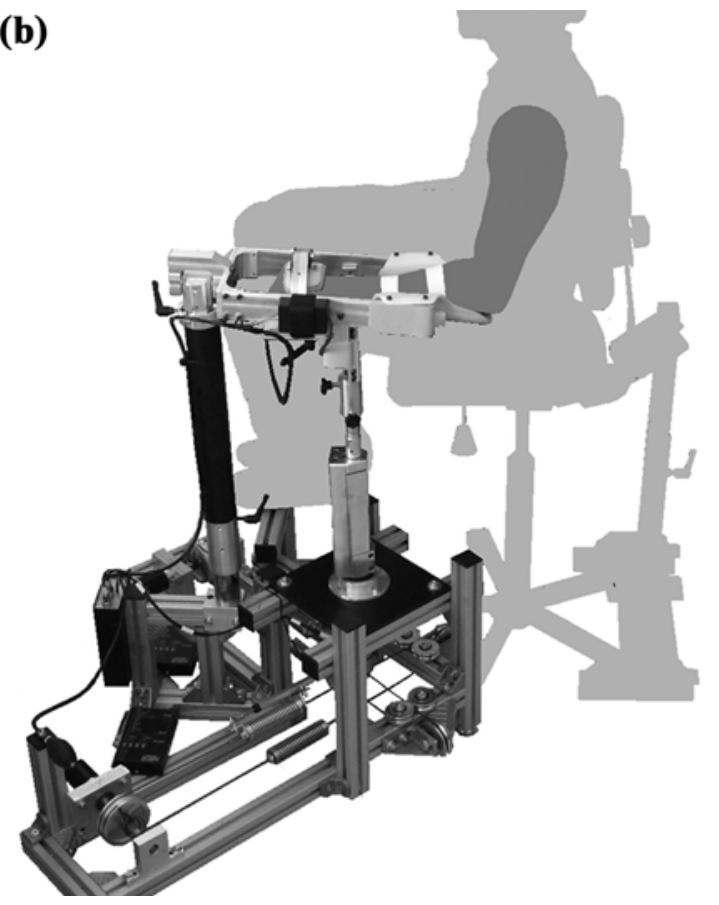

Figure 1.

(a) Mechanical structure of Universal Haptic Drive (UHD) and (b) revised pantograph kinematic structure of Universal Haptic Pantograph (UHP). In UHD design, posterior-mounted forearm support was used to fix forearm when in Wrist mode and was removed for Arm mode, whereas in revised UHP design, configurable pantograph mechanism can be used to support forearm during all device modes without removal and, furthermore, enables six additional device modes.

lower end of each vertical bar is attached to a common base by a multiaxis joint, while the top of the two vertical bars are connected by the Transverse Assembly. The Actuated Bar, the bar closest to the user, extends down through a spherical joint to a distance below the base plate where it is actuated by the orthogonal set of SEAs. A number of components are linked in series along the pantograph mechanism, above the SEA attachment to the Actuated Bar (Figure 2(a)). Starting from the base closest to the user, the components are a-

1. Passive spherical joint.

2. Sliding mechanism and spring suspension system that enables adjustable gravitational support of the arm.

3. 2-DOF universal joint that can be locked (joint lock A).

4. Set of low-cost force sensors.

5. Passive 1-DOF wrist joint.

6. Forearm orthoses.

7. Hand attachment and wrist orthoses.

8. 1-DOF revolute joint for wrist pronosupination.

9. 1-DOF revolute joint that can be locked (joint lock B).

10. Lightweight structural tubing.
11. 2-DOF universal joint that can be locked (joint lock $\mathrm{C}$ ). Components 1 through 4, 5 through 8, and 9 through 11 are distributed, respectively, along the Actuated Bar, Transverse Assembly, and Parallel Bar.

\section{Actuated Bar}

The Actuated Bar uses the same spherical base joint, component 1 shown in Figure 2(a), as used in the previous UHD system (Figure 2(b)). The spring suspension system, component 2 shown in Figure 2(a), is composed of a lightly damped compression spring $(3.19 \mathrm{~N} / \mathrm{mm})$ that is compressed when a sliding mechanism shortens. The resting length of the spring relative to the sliding mechanism can be easily adjusted using a rope, pulley, and cam cleat system (Figure 3). As a result, the amount of gravitational support given to the user in the neutral position of the device can be varied and is calculated as the product of the spring stiffness and the distance translated by the upper sliding part, shown in Figure 3. The translated distance of the part is measured by the combination of a reflective surface mount optimal encoder (Agilent Technologies; Santa Clara, California) and a code strip that are 
(a)

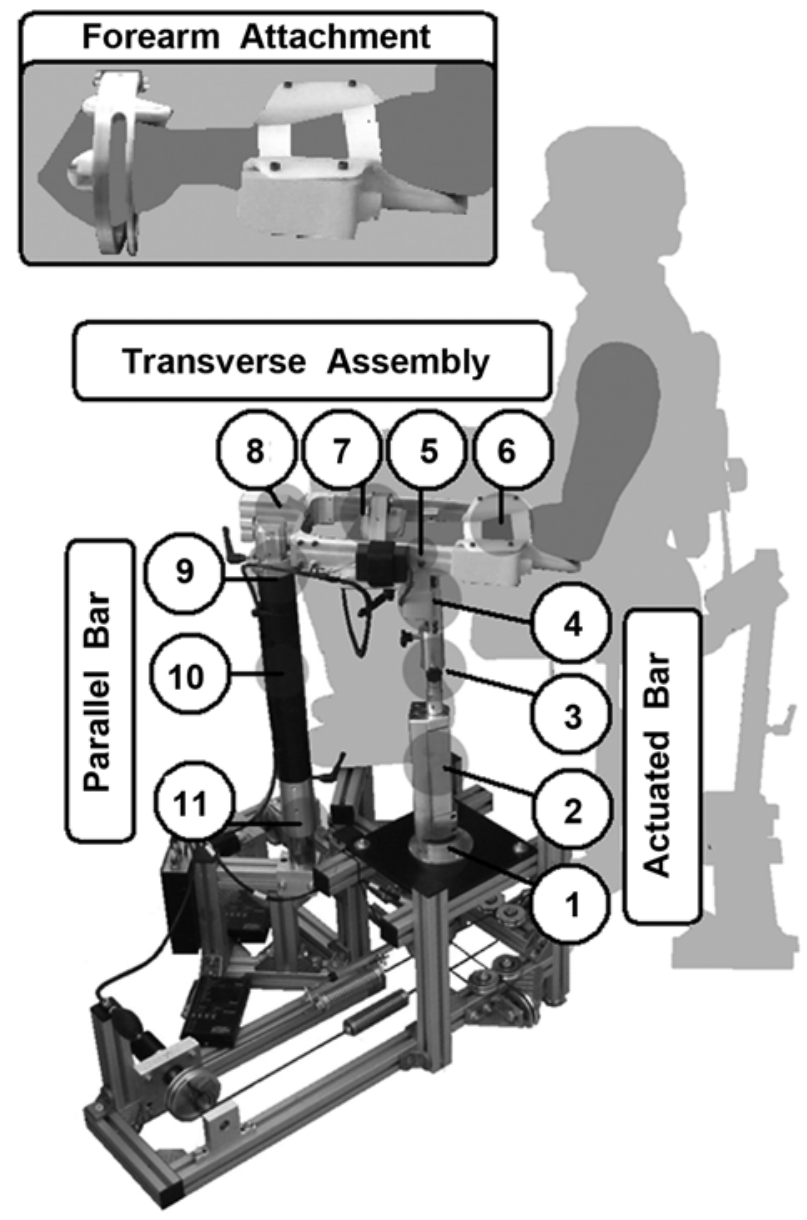

(b)

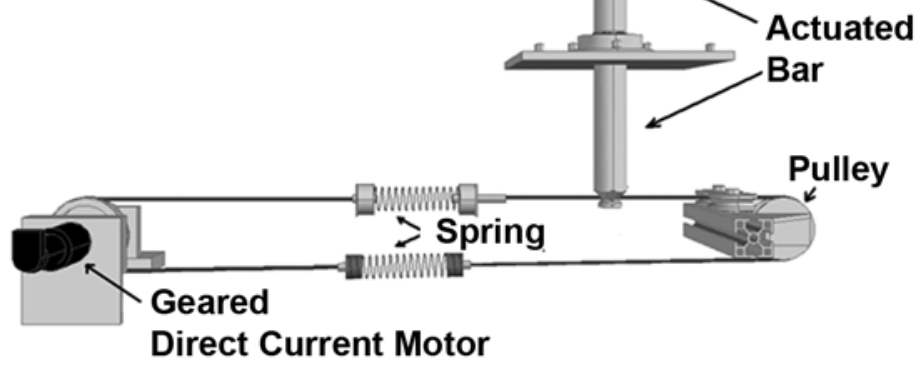

(c)

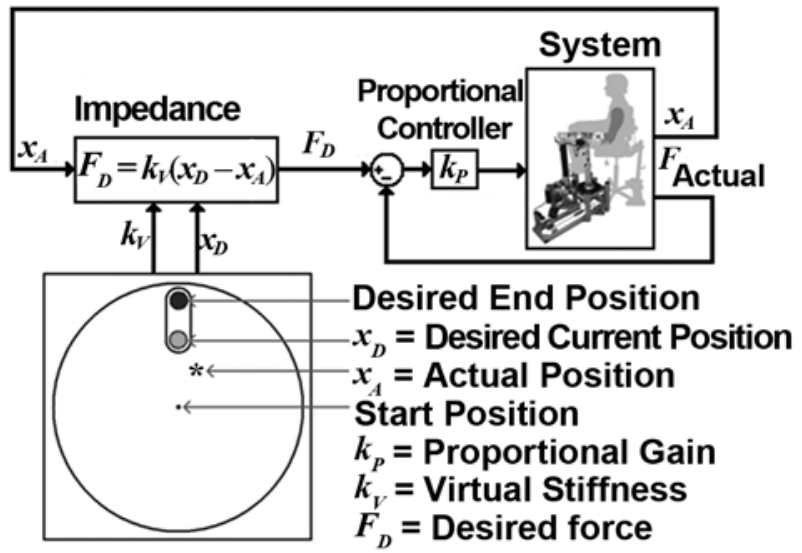

Virtual Reality Environment (Computer Screen)

Figure 2.

Overall system: (a) Pantograph mechanism consists of (1) passive spherical joint, (2) spring suspension system, (3) passive lockable 2-degree-offreedom (DOF) universal joint A, (4) low-cost 2-axis force sensors, (5) passive 1-DOF wrist joint, (6) user forearm attachment, (7) user wrist attachment, (8) passive forearm pronosupination joint, (9) passive lockable 1-DOF revolute joint lock B, (10) lightweight structural tubing, and (11) passive lockable universal joint lock C. (b) Series elastic actuator-based drive. (c) Impedance-based control scheme and training scenario graphical user interface.

placed along the inner surface of the upper and lower sliding parts, shown in Figure 3, respectively.

A universal joint below the wrist, component 3 in Figure 2(a), allows the wrist motion to be locked or unlocked with lock A (Figure 4(a)). When joint lock $A$ is used with joint locks B and C (Figure 4(a)), the prescribed motion of the arm and hand is altered for different training tasks. A set of low-cost force sensors, component 4 in Figure 2(a), with a maximum force range of $\pm 100 \mathrm{~N}$ was mounted in an orthogonal configuration between joint lock $\mathrm{A}$ and the handle, with the function of measuring user interaction forces in the plane of sensor motion.

\section{Transverse Assembly}

The framework of the Transverse Assembly is composed of a U-shaped structure that surrounds the hand and forearm and functions as a 2-DOF wrist gimbal, where one of the two axes is aligned with the longitudinal axis of the forearm, component 8 in Figure 2(a), allowing pronosupination. The second transverse axis is a 1-DOF wrist axis orthogonal to the plane defined by the vertical bars of the pantograph mechanism and, therefore, depending on the orientation of the handgrip, enables either wrist flexion/extension or radial/ulnar deviation, component 5 in Figure 2(a). 
(a)

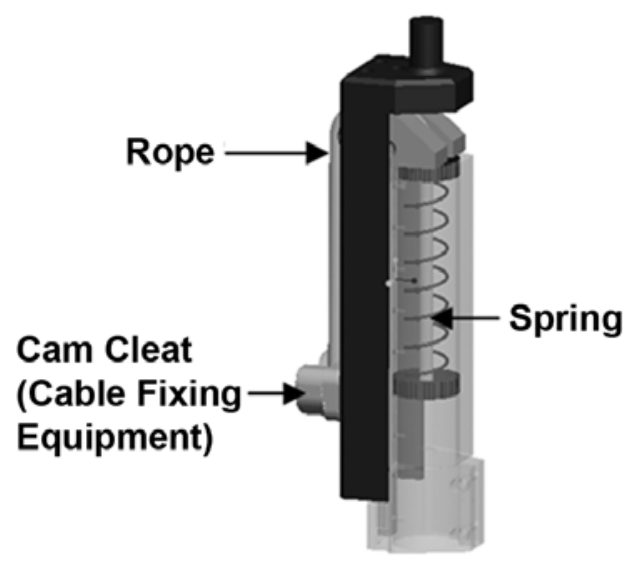

(b)

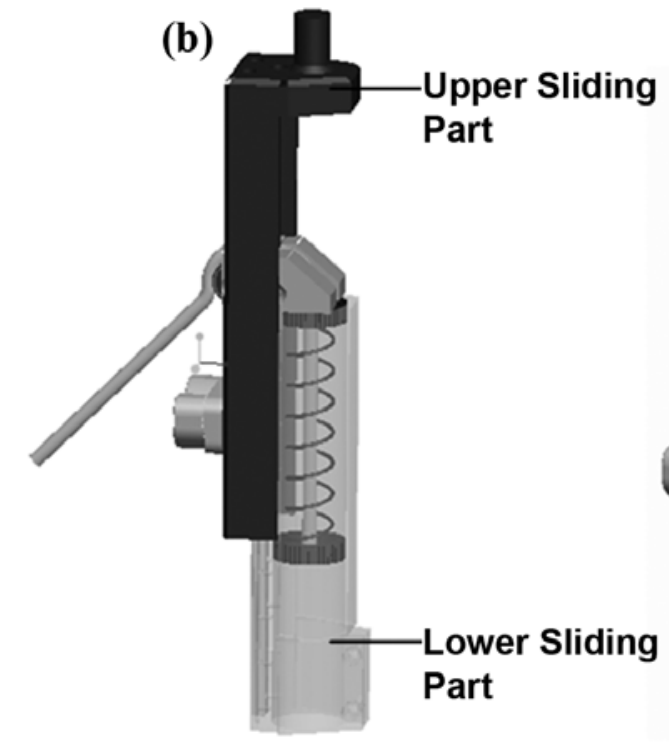

(c)

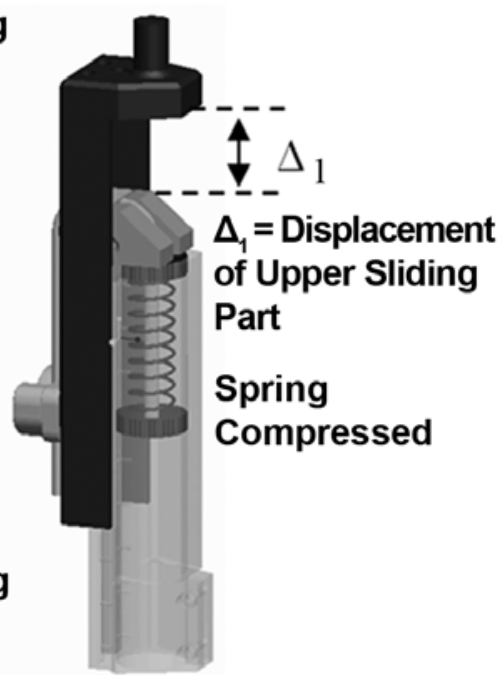

Figure 3.

Spring suspension system and its adjustment (lower sliding part is shown in transparent to expose spring): (a) Step 1-initial state with rope fixed to cam cleat, (b) Step 2 - unfixed rope and raised upper sliding part to set level of spring support, (c) Step 3-rope fixed to cam cleat with upper sliding part allowed to lower to new neutral position of spring suspension. Spring will have some initial compression due to weight of parts (e.g., Transverse Assembly) connected in series above upper sliding part. Note that for given set position of rope, gravitational support varies linearly with $\Delta_{1}$.

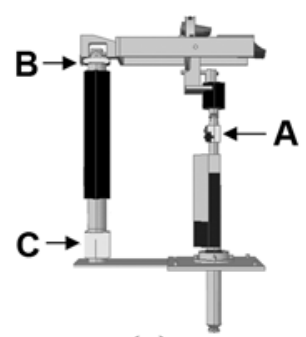

(a)

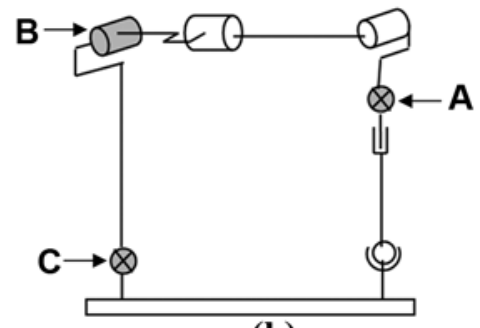

(b)
Figure 4.

Pantograph mechanism with variable structure (joints $\mathrm{A}, \mathrm{B}$, and $\mathrm{C}$ may be locked or unlocked): (a) 3-dimensional computer-aided design drawing and (b) schematic drawing.

The user's arm is thereby attached to the device at two locations; the user's forearm is attached to the Transverse Assembly by a forearm orthosis, component 6 in Figure 2(a), and the user's hand is attached to the Actuated Bar by a handle and wrist orthosis, component 7 in Figure 2(a). The handle position adjusts for aligning the 1DOF transverse device wrist, component 5 in Figure 2(a), with the user's wrist joint and also adjusts in orientation for setting the neutral pronosupinated position of the user's forearm. The handgrip orientation can be adjusted such that the neutral grip is achieved under varying degrees of pronosupination $\left( \pm 45^{\circ}\right)$.

\section{Parallel Bar}

The Parallel Bar provides two modes of support to the forearm by means of two configurable joints (B and $\mathrm{C}$ in Figure 4(a)) for transferring either structural rigidity or prescribed motion. The upper joint (B) is composed of a 1-DOF revolute joint, component 9 in Figure 2(a), and is oriented parallel to the transverse wrist axis and the Transverse Assembly to remain parallel to the floor. The lower joint (C) is composed of two universal joints, component 11 in Figure 2(a), that can be locked to prevent translation of the Transverse Assembly, whereas joint lock B prevents its rotation. The majority of the bar itself is composed of a lightweight aluminum structural tubing, component 10 in Figure 2(a).

A schematic representation of the UHP (Figure 4(b)) illustrates the system's articulations, composed of a 3-DOF spherical joint, two universal joints, three 1-DOF revolute joints, and one 1-DOF prismatic joint. Highlighted in Figure 4, two universal joints (A and $\mathrm{C}$ ) and one revolute joint lock (B) are equipped with joint locks that can be selectively engaged or disengaged for configuring the structure's kinematic workspace into one of five distinct 
system configurations. Additionally, each mode of operation can be shown to possess fewer than 4 DOFs, reducing the number of actuators needed and simplifying the system control. With simple end effectors such as a secondary handle included, the system's lock configurations yield a minimum of eight kinematic modes for various aspects of shoulder, elbow, and wrist training with a system. The system lock configurations and kinematic modes are described in detail in the following sections.

\section{System Lock Configurations}

As illustrated in Figure 4(a), the pantograph mechanism of the UHP is composed of 7 links and 7 joints, totaling 11 axes of motion. For reasons of stability, the system (and/or environment) must be capable of applying a restoring force against user movement in any system configuration. In the case of the UHP, the SEAs provide 2 active DOFs for stabilizing user movement and the spring suspension system provides 1 DOF. As a result, one can state that a requirement for system stability in the UHP is that all operational modes of the device are limited to a maximum of 3 DOFs. The number of DOFs of the UHP system in each configuration of joint locks can be determined with Grubler's formula as

$$
F=6(l-n-1)+\sum_{i=1}^{n} f_{i},
$$

where $F$ is the total number of DOFs of the mechanism, $l$ is the number of links (including the base), $n$ is the number of system joints, and $f_{i}$ is the number of DOFs associated with the ith joint [28]. Following this equation, the total number of DOFs for the unlocked UHP system can be computed as $F=6(7-7-1)+11=5$ DOFs.

Alternatively, one may conceptually derive the number of system DOFs by considering the mechanical structure not as one parallel mechanism but rather as two serial mechanisms connected at the handle. In this case, the system is composed of one 5-DOF (passive) mechanism and one 6-DOF (2 active and 4 passive) mechanism. Because the passive mechanism has 5 DOFs, it can achieve a given handle position and orientation in only one distinct pose. The actuated mechanism, however, by virtue of its sixth DOF, can reach positions within its workspace in more than one orientation. In this way, the 6-DOF mechanism can move freely to connect at the handle to the passive mechanism and, thereby, be constrained to the same DOFs allowed by the passive 5 DOFs.
With a combined parallel system, any new constraint added to either of the two serial mechanisms will add the same constraint to the combined 5-DOF system. Therefore, the three joint locks (two universal joint locks and one 1-DOF revolute joint lock) sufficiently constrain the system fully. From this understanding, the analysis of configuration-specific DOFs becomes trivial, but for clarity, they will be determined with the use of Grubler's formula.

Considering the 5-DOF mechanism of Figure 4, five joint lock combinations yield system work spaces appropriate for haptic training in the shoulder, elbow, and wrist. Illustrated in Table 1, the combinations of engaged (locked - shown in black) and disengaged (unlockedshown in gray) joints are named according to the locks that are engaged in each combination (e.g., configuration $\mathrm{BC}$ refers to the configuration where $\mathrm{B}$ and $\mathrm{C}$ are locked and $\mathrm{A}$ is unlocked). The five combinations are $\mathrm{ABC}, \mathrm{BC}$, $\mathrm{A}, \mathrm{AB}$, and $\mathrm{AC}$. Recall that joint locks $\mathrm{A}, \mathrm{B}$, and $\mathrm{C}$ represent 2-, 1-, and 2-DOF joints, respectively, so configuration $\mathrm{BC}$ produces a system having five links, five joints, and eight axes of motion yielding a system workspace of $F=6(5-5-1)+8=2$ DOFs.

\section{Kinematic Modes}

As just described, the developed UHP can be configured into one of five workspace configurations. In general, the modes are designed to target either the shoulder or elbow for placement of the hand or the wrist for orientation of the hand. Furthermore, the device workspace for shoulder and elbow training is designed to train combinations of shoulder and elbow extension to target abnormal muscle synergies in the patient population and enlarge the distal workspace. As a result, the current workspace configurations may not be suitable for certain proximal ADL tasks such as eating or facial hygiene. However, interface add-ons could be developed for proximal work spaces, adding modes for hygiene tasks or other tasks that combine aspects of shoulder, elbow, and wrist into specific coordinated tasks.

From the five workspace configurations, the number of end-effector-based work spaces can be significantly increased $(>5)$ with the addition of external end-effector add-ons. For illustration in this article, considering the inclusion of a end-effector add-on as a cantilevered handle, the system can be configured into one of eight mechanical work spaces, or kinematic modes. The eight kinematic modes available (including a cantilevered-handle add-on in some modes) are Arm, Wrist, ISO (isometric) 1, ISO 2, Reach, Lift 1, Lift 2, and Steer. 
Table 1.

Modal lock configurations (black $=$ locked joint, gray $=$ unlocked joint).
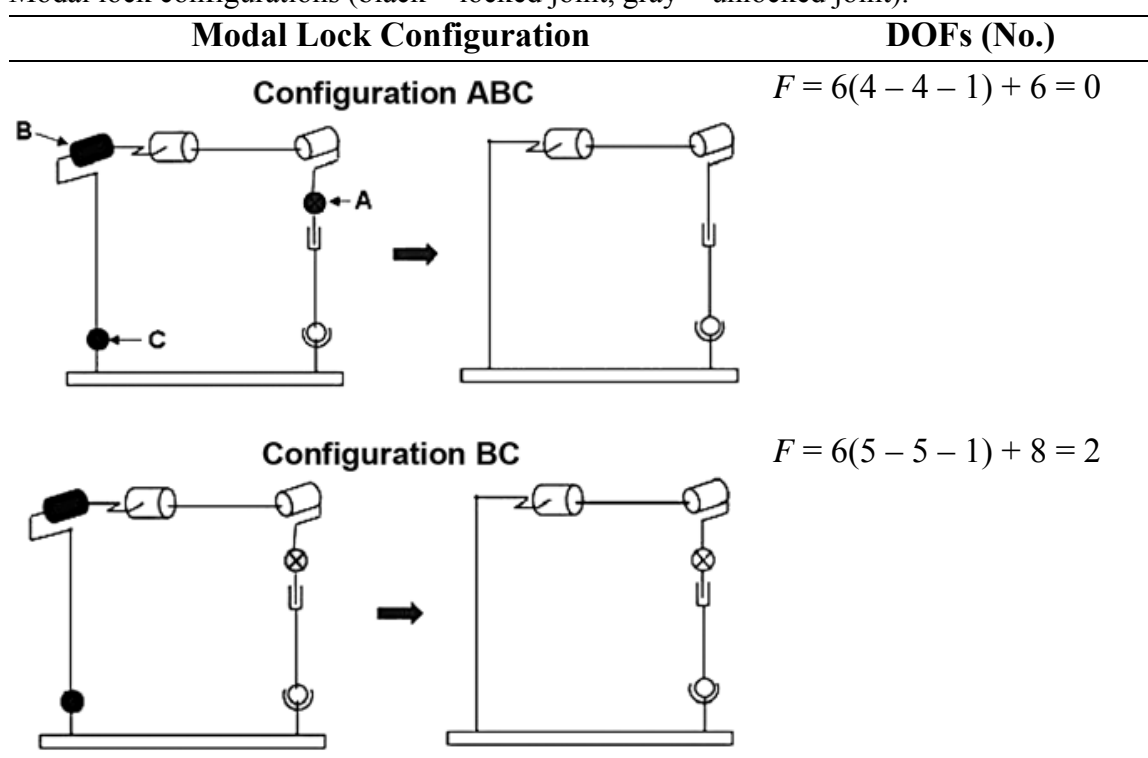

$F=6(5-5-1)+8=2$

Only 2-DOF universal joint lock A below wrist is unlocked, allowing either wrist F/E, radial/ ulnar deviation, and pronosupination, depending on orientation of user handle.

All joint locks are engaged, constraining system to single-point workspace for isometric measurement and strength training of wrist $\mathrm{F} / \mathrm{E}$, radial/ulnar deviation, and pronosupination, depending on orientation of user handle.
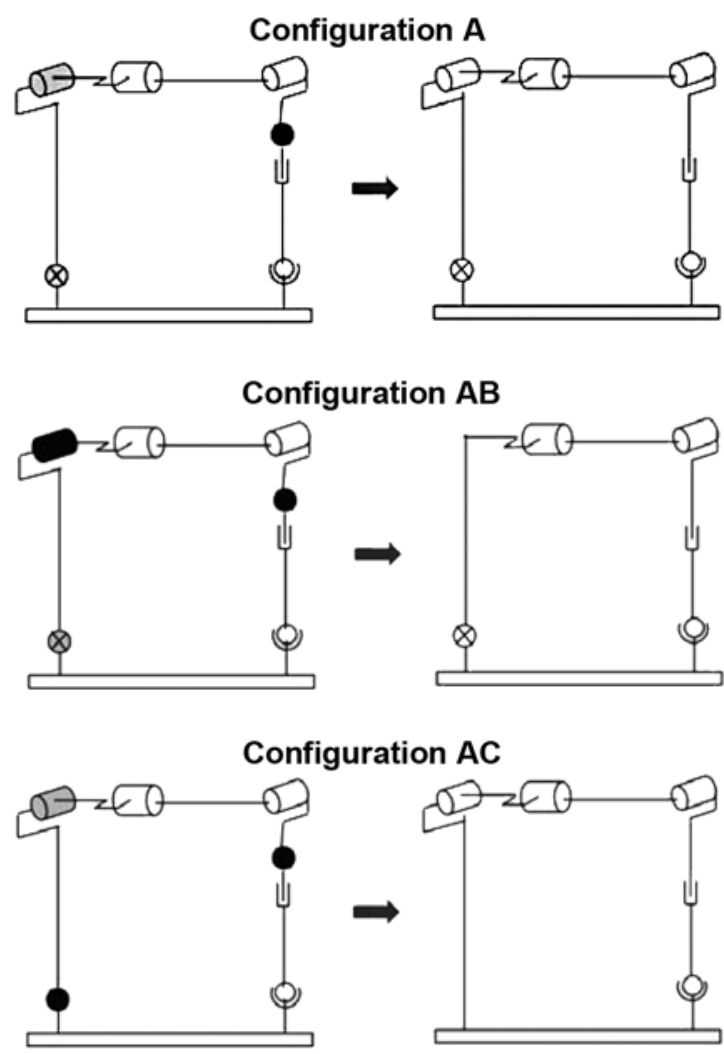

$$
F=6(6-6-1)+9=3
$$

Configuration A constrains relative movement between device handle and Actuated Bar. Configuration is suitable for planar arm training if user exerts no vertical force or for 3-D arm training under various loading conditions of spring suspension system.

$$
F=6(5-5-1)+8=2
$$

Only 2-DOF universal joint lock $\mathrm{C}$ at base of Parallel Bar is unlocked, prescribing coupled motion between fore and aft pitch of Parallel Bar and elongation of sliding mechanism. Additionally, for a given sliding mechanism elongation, pantograph can tilt laterally to motion limits of spherical joint.

$$
F=6(5-5-1)+7=1
$$

Joint locks $\mathrm{A}$ and $\mathrm{C}$ produce a workspace that gives user a high mechanical advantage over actuation system, significantly reducing force transmission of actuators. Kinematically, system in this configuration enables vertical weight lifting against gravity for low-level forces under variable gravitational support from spring suspension system.

\section{Arm Mode-Lock Configuration A}

In the first mode or Arm mode, with the arm attached to the wrist and forearm orthoses (Figure 5), a subject holding the handle can perform quasiplanar reaching movements, as shown by arrows in Figure 5. Here, the expression quasiplanar means that the patient's hand is constrained 
to movements along the surface of a partial sphere. The workspace is limited by the $15^{\circ}$ maximal lateral tilt of the Actuated Bar. With sufficient support from the spring suspension system, the user can also move within a limited ROM perpendicular to the spherical surface by moving the sliding mechanism. The movement prescribed by the workspace in the Arm mode is similar to what is required to reach or move objects on a table, desk, or countertop.

\section{Wrist Mode-Lock Configuration BC}

In the second mode or Wrist mode (Figure 6), with the arm attached to wrist and forearm orthoses, the entire Parallel Bar is mechanically locked (Figure 6(a)) so that a rigid structure is created that constrains the center position of the wrist gimbal. The handle bar (i.e., the segment of the Actuated Bar above the unlocked universal joint A) rotates relative to the Actuated Bar in 2 DOFs, inducing an amplified motion in the handle bar. Given the segment lengths shown in Figure 5, a rotation of $15^{\circ}$ in the Actuated Bar produces a $45^{\circ}$ rotation in the handle. In this mode, a subject can perform 2-DOF motions of the wrist, where the first DOF is always supination/pronation of the forearm (Figure 6(b) and 6(c)) and the second DOF depends on the orientation of the handle. When the handle is oriented horizontally, the second DOF is extension/flexion (Figure 6(d) and 6(e)), and when the handle is oriented vertically, the second DOF is radial/ulnar deviation (Figure 6(f) and 6(g)), respectively. Comparisons between achievable ROM of the UHP in Wrist mode and the human wrist have been previously shown [27]. Note that in this mode, a change in length of the segment below universal joint lock A occurs because of the fixed position of the wrist gimbal. This change is compensated by elongation of the sliding mechanism in the spring suspension system. The resulting movement of the user's wrist may be similar to what is required to perform the wrist-orienting aspect of activities such as operating a mouse, pouring from a bottle, brushing the teeth, or stirring a pot.

\section{ISO 1 Mode-Lock Configuration ABC}

In the third mode or ISO 1 mode, with the arm attached to wrist and forearm orthoses, joints $\mathrm{A}, \mathrm{B}$, and $\mathrm{C}$ constrain all DOFs of the device. In this mode, isometric forces of the wrist can be measured from the force sensors in all the directions of device motion that are allowed under the Wrist mode. The isometric measures obtained can be used for isometric strength training or for user-specific calibration of the system. (a)

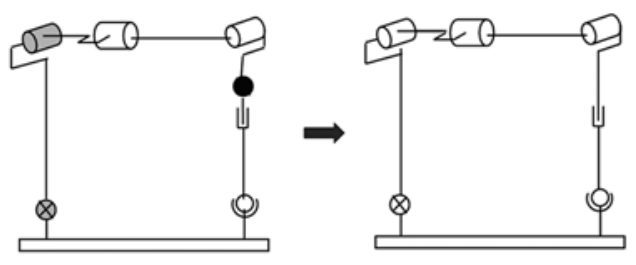

(b)
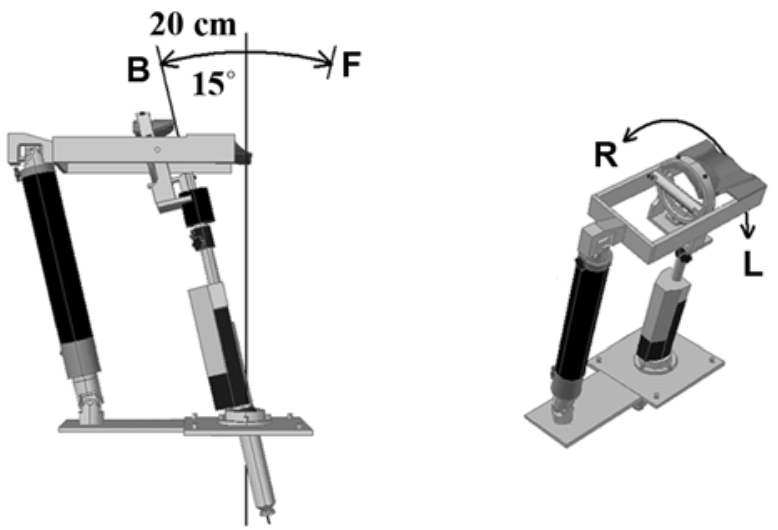

(c)

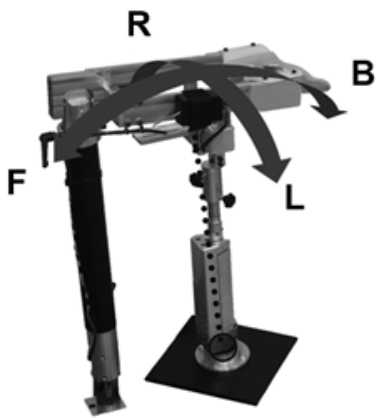

Figure 5.

Arm mode: (a) Schematic drawing of lock configuration A (refer to Table 1), (b) backward (B)-forward (F) and right (R)-left (L) movement directions shown in 3-dimensional computer-aided design drawings, and (c) depiction of spherical surface motion with actual Universal Haptic Pantograph.

\section{ISO 2 Mode—Lock Configuration AC}

The fourth mode is the ISO 2 mode, with the arm attached to wrist and forearm orthoses. With the joint lock B disengaged, varying degrees of arm elevation force can be exerted and measured while isometric wrist exercises are performed. ISO 2 is a more advanced mode of training that requires simultaneous control of both shoulder and wrist motor units.

\section{Reach Mode-Lock Configuration AB}

In the fifth mode or Reach mode, with the hand holding an add-on cantilevered handle mounted horizontally 
(a)
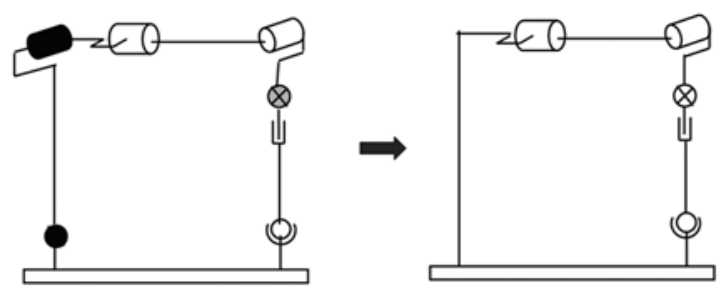

(b)
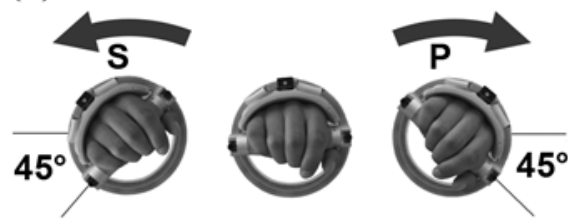

(c)

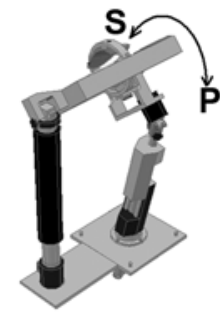

(d)

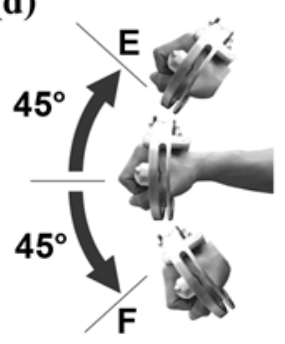

(f)

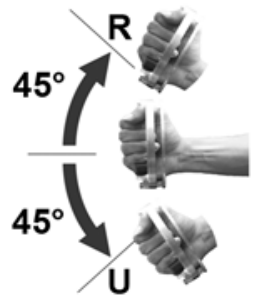

(e)

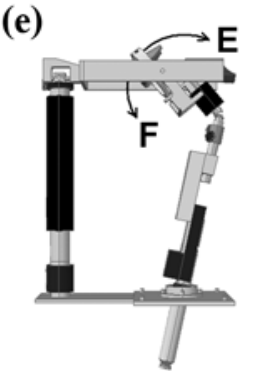

(g)

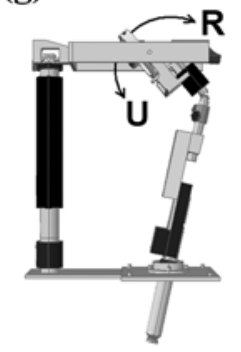

Figure 6.

Wrist mode: (a) Schematic drawing of lock configuration BC (refer to Table 1), (b) pronation (P)/supination (S), (c) movement of Universal Haptic Pantograph (UHP), (d) flexion (F)/extension (E), (e) movement of UHP under F and E, (f) radial (R)/ulnar (U) deviation, and (g) movement of UHP under R and $\mathrm{U}$.

(posterior aspect), the trajectory of the reach task is altered from that of the Arm mode by the additional engagement of joint lock $\mathrm{B}$. This joint lock imposes a $90^{\circ}$ constraint between the Parallel Bar and Transverse Assembly (Figure 7(a)), and the resulting motion of the handle is a combination of the Parallel Bar's universal joint $\mathrm{C}$ and the Actuated Bar's sliding mechanism. With the addition of a simple handle extending horizontally toward the user from the posterior aspect of the Trans-

verse Assembly, the user performs an upward and leftright reach task. The work space of the exercise is constrained to the upper-frontal aspect of a large spherical surface, where its radius is defined by the distance from the attached handle to universal joint $\mathrm{C}$ (Figure 7(b)). The motion is therefore similar to activities such as reaching for a high drawer or cupboard or moving objects from one side of the cupboard to the other.

\section{Lift 1 Mode-Lock Configuration BC}

The sixth mode, Lift 1 mode, is operated with the hand holding an add-on cantilevered handle mounted horizontally (posterior aspect). As illustrated in Figure 8, the prescribed motion of the added handle in Lift 1 mode, lock configuration BC (Figure 8(a)), is constrained to a 2-DOF spherical surface, the radius of which is defined by the distance from the handle to the transverse axis of the wrist (Figure 8(b)). The motion is primarily in a vertical reach direction and is therefore similar to activities such as reaching and replacing objects from a high shelf, putting on a hat, or lifting a lid.

\section{Lift 2 Mode-Lock Configuration AC}

The seventh mode or Lift 2 mode, with the hand holding an add-on cantilevered handle mounted horizontally (posterior aspect), is a second method of vertical lift training, where the motion is constrained to a 1-DOF trajectory. In this mode, the user lifts upward on the posterior handle under gravitational assistance from the spring suspension system. Lock configuration AC may particularly benefit users with excessively low strength in the vertical direction because the amount of support provided can be adjusted as needed. The Lift 2 mode primarily differs from the Lift 1 mode in that Lift 1 allows motion training under larger resistive and assistive forces provided by the (a)

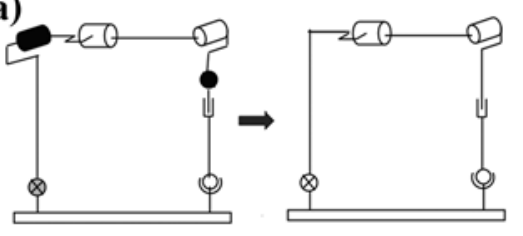

(b)

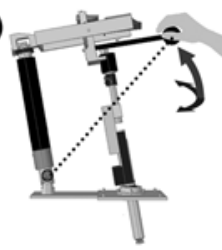

Figure 7.

Reach mode; left/right and up/forward and down/backward movement in 2 degrees of freedom: (a) Schematic drawings of lock configuration AB (refer to Table 1) and (b) 3-dimensional (3-D) computer-aided design drawing. Dotted line in (b) represents radius of 2-D motion. 
(a)

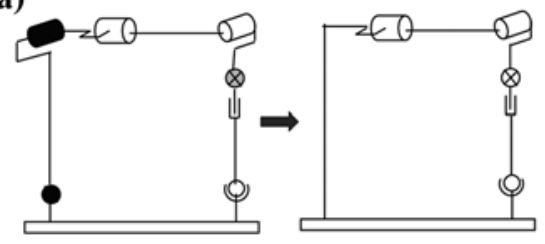

(b)

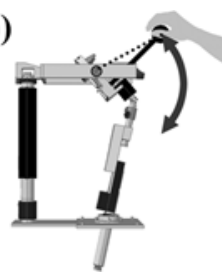

Figure 8.

Lift 1 mode: (a) Schematic drawings of lock configuration BC (refer to Table 1) and (b) 3-dimensional (3-D) computer-aided design drawing showing quasivertical movement in 1 degree of freedom. Dotted line in (b) represents radius of 1-D motion.

2-DOF SEAs, whereas Lift 2 assists the user through the passive spring suspension system.

\section{Steer Mode—Lock Configuration BC}

The eighth mode is the Steer mode, with holding add-on cantilevered handle mounted vertically (superior aspect), and is for forward and lateral-elevated arm training. In an eighth mode of the device, the same joint configuration used in both Wrist and Lift 1 modes (lock configuration $\mathrm{BC}$ ) is combined with the handle add-on attached vertically. By grasping the handle positioned above the device's pronosupination axis, the user can perform reaching or steering movements on the surface of a sphere, where its radius is defined by the distance above the pronosupination axis. In this mode, the user trains on the forward and lateral-elevated arm control. The mode can be used to train activities such as operating a steering wheel, manual transmission gearshift, or other lever arm.

\section{Actuation and Control}

A SEA system was previously developed for the UHD and described by Oblak et al. [27]. Two such SEAs are placed perpendicularly below at the base of the Actuated Bar. Each SEA assembly consists of a geared direct current motor with encoder connected in series with the Actuated Bar with a wire rope, extension spring, and a pulley as shown in Figure 2(b). The SEA components have been used for three primary reasons: first, to solve the force-coupling problem near device ROM limits, resulting from the geometric design of the UHD actuation; second, for the many benefits that they provide in force control; and third, for their inherent safety in human machine interfaces. All three reasons are investigated and presented in detail in previously published work on the UHD [27]. We determined spring stiffness in the actuation experimentally to provide sufficient bandwidth $(\sim 1 \mathrm{~Hz})$ for rehabilitation with the UHD, that is, under the slow movements expected during training.

We implemented impedance-based force control in each actuated DOF for all training modes as shown in Figure 2(c). A simple visual interface to the virtual realitytraining environment displays a user's actual, current desired, and final desired positions. A user's initial position always begins at the center of a bounding circular work space, while the desired current and final (end) position markers are shown to the user by green and red (shown as gray and black circles in Figure 2(c)), respectively, and the user's actual position is shown by a blue asterisk (shown as black in Figure 2(c)). A restoring force is calculated from a selected impedance parameter $\left(k_{V}=\right.$ virtual stiffness) and the measured deviation between the user's desired and actual position. Based on this measured error, a restoring force assists the user in tracking the desired movement trajectory. If selected virtual stiffness $k_{V}$ is low, the restoring force is small even though deviations between a user's desired and actual positions may be large, resulting in a low-impedance environment in which user-in-charge oriented exercises are performed. On the other hand, in a high-impedance environment, where $k_{V}$ is high, the restoring force is large if the actual and desired positions do not match. In that case, the restoring force does not allow the user to move freely, and therefore, a robot-in-charge mode occurs. The restoring force is compared with a feedback signal measured by means of the force sensor on the handle bar, which thereby closes the force loop.

\section{RESULTS: CLINICAL EVALUATION}

We developed the proposed pantograph mechanism from the basis of the first UHD design. A set of three preliminary case studies were performed with three subjects of different FMA and WMFT scores (Table 2). Of the resulting eight kinematic modes of the UHP, we selected the most appropriate modes for each patient and used them to evaluate the performance of the redesigned UHP. These modes were the Wrist mode for subject A and the Arm and Reach modes for subjects B and C. Other modes of the device were tested separately but not included in the case studies. For all subjects, training was divided into three sessions of 7 minutes each and performed on consecutive workdays such that each subject trained for approximately 30 minutes a day (including 
JRRD, Volume 48, Number 4, 2011

Table 2.

Impairment and initial assessment data for three subjects.

\begin{tabular}{|c|c|c|c|c|}
\hline Subject & $\begin{array}{c}\text { Description } \\
\text { of Chronic } \\
\text { Hemiparesis }\end{array}$ & $\begin{array}{c}\text { Period } \\
\text { After } \\
\text { Stroke }\end{array}$ & $\begin{array}{l}\text { Initial } \\
\text { FMA }\end{array}$ & $\underset{\text { WMFT }^{*}}{\text { Initial }}$ \\
\hline A & $\begin{array}{l}\text { Right side with } \\
\text { limited movement } \\
\text { abilities especially } \\
\text { in wrist }\end{array}$ & 10 years & 32 & 36 \\
\hline B & $\begin{array}{l}\text { Left side with lim- } \\
\text { ited movement } \\
\text { abilities in shoul- } \\
\text { der, elbow, and } \\
\text { wrist }\end{array}$ & 6 months & 8 & 17 \\
\hline $\mathrm{C}$ & $\begin{array}{l}\text { Left side with lim- } \\
\text { ited movement } \\
\text { abilities in shoul- } \\
\text { der, elbow, and } \\
\text { wrist }\end{array}$ & 6 months & 37 & 33 \\
\hline
\end{tabular}

${ }^{*}$ Scores measured at beginning of training.

FMA $=$ Fugl-Meyer Motor Assessment, WMFT $=$ Wolf Motor Function Test.

pauses between sessions). For the Wrist mode training, the three training sessions corresponded to training flexion-extension, radial-ulnar deviation, and pronosupination, and for shoulder and elbow training, the training sessions were composed of the Reach mode and two variations of the Arm mode, one with the standard visual interface (as described by Oblak et al. [27]) and one with the same task performed within a virtual environment. We used approximately 30 minutes for the three training sessions to reduce the possibility of fatigue in the subjects and also the unreliable effects that fatigue may introduce in the training results.

For subject A, the training was done in the Wrist mode based on the degree of limited movement ability in the wrist. The objective of the movement training was to track target points, which were composed of 2-DOF motions of the wrist, including radial/ulnar deviation, pronosupination, and flexion/extension. Depending on the orientation of the handle and the visual interface, the subject was requested to perform tasks involving primarily one of the three wrist motions. Training sessions for subject A were performed on 18 consecutive days composed of three 7-minute sessions. In the first 2 minutes of training, the subject was completely passive while we set up the UHP to provide 100 percent support to show the subject the desired task. One-hundred percent support means that the value of virtual stiffness that can be achieved by the UHP was set to the maximum [27], and the subject was therefore guided through the task by the robot. During the next 5 minutes, the UHP offered the subject assistive force, as needed, to successfully track the target position. In this way, the level of support offered to the subject was automatically reduced over the training sessions as the subject improved. FMA and WMFT scores were assessed 2 weeks before training, during the training period (including start and end), and 2 and 4 weeks after the cessation of training as shown in Figure 9.

For subject B, training sessions were performed in both the Arm and Reach modes. In the Arm mode, the training objective was to track the target position in eight different directions within a quasiplanar movement as described by Oblak et al. [27]. In particular, the subject was asked to keep the elbow elevated to impose a vertical load on the shoulder (facilitated by the spring suspension system), and the vertical load was monitored. In the Reach mode, the objective was to track the target position in three different directions of forward/lateral reach movements. The training for subject B was performed on 14 consecutive days, composed of three 7-minute sessions, divided into the same 2-minute full and 5-minute partial support intervals that were completed with subject A. We assessed FMA and WMFT scores 2 weeks before training, during the training period, and 2 weeks after the training, as shown in Figure 10. In particular, Figure 11 illustrates UHP progression of reduced support as training sessions progressed and subject mobility improved.

The Arm and Reach modes were also selected for subject $\mathrm{C}$, and hence, the same training procedure was followed as performed with subject B, except for the number of training sessions performed. Subject $\mathrm{C}$ participated in nine training sessions and canceled further training for personal reasons unrelated to the training procedure. FMA and WMFT scores were measured 2 weeks before training, during the training period, and 3 weeks after training as shown in Figure 12.

\section{DISCUSSION AND CONCLUSIONS}

This article presents a novel pantograph mechanism combined with a spring suspension system for comprehensive rehabilitation training of the shoulder, elbow, and wrist. This third-generation system, built upon a previous two-mode UHD system, enhances the number of system configurations from two to five and the number of training modes from two to eight, increasing the ease of mode changes and improving the method of providing gravity 


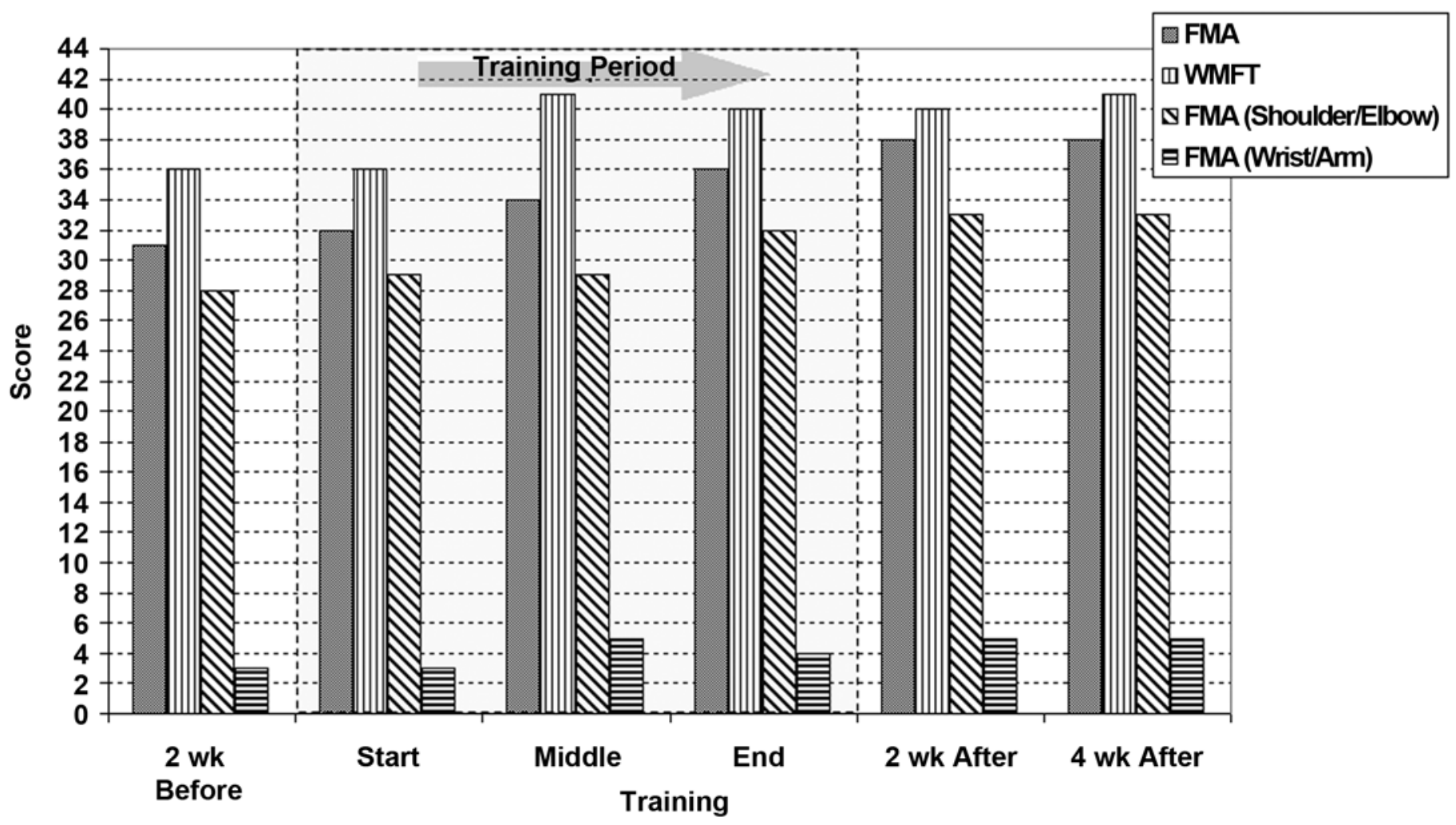

Figure 9.

Fugl-Meyer Motor Assessment (FMA) and Wolf Motor Function Test (WMFT) scores for subject A: Wrist-mode training.

compensation that facilitates variable loading/support of the arm weight applied to the shoulder.

From a kinematic analysis of the developed pantograph mechanism, the number of device DOFs resulting from each of the five joint lock combinations has shown to yield a system having 3 DOFs. As a result, the same two active SEA components used in the first UHD mechanism are in the redesigned pantograph mechanism that is capable of actuating the various system DOFs in all eight training modes, including arm-reaching and -lifting, wrist-orientation, and steering tasks. Kinematic modes Arm, Reach, Lift 1, and Lift 2 are primarily shoulder/ elbow training modes that allow strength and motorcontrol training for general reach tasks in the forward, lateral, and vertical directions and are designed to simulate such tasks as reaching or moving objects on a table or high shelf or in a cupboard. Wrist and ISO 1 modes are wrist-training modes that allow strength and motorcontrol training for wrist orientation tasks, designed to simulate general object orienting such as operating a mouse, pouring from a bottle, brushing the teeth, or stirring a pot. ISO 2 and Steer modes are examples of training modes that allow combined training for the shoulder, elbow, and wrist under specific constraints for performing wrist manipulation tasks under progressive abduction loading in the ISO 2 mode or for car-steering or shifting tasks in the Steer mode. The mechanism design thereby demonstrated a high suitability for training most upper-limb ADL tasks involving hand positioning and orientation.

The performance of the mechanism, driven by current SEA components (high-cost precision motors and gear heads, and low-cost springs, cables, and pulleys), is similar in all operational modes while a control scheme and set of gains are used. This finding means that a single device with minimal setup changes can be used to treat a variety of upper-limb impairments such as stroke, traumatic brain injury, or other direct trauma to the arm that commonly afflict veterans. The current relatively highcost motors used in the UHP's SEAs, with their high backdrivability and low backlash, will be substituted for alternative low-cost motors for improving the affordability of the final system. From initial analyses and evaluation with low-cost replacement motors, we found that optimizing SEA damping could produce a system that 
JRRD, Volume 48, Number 4, 2011

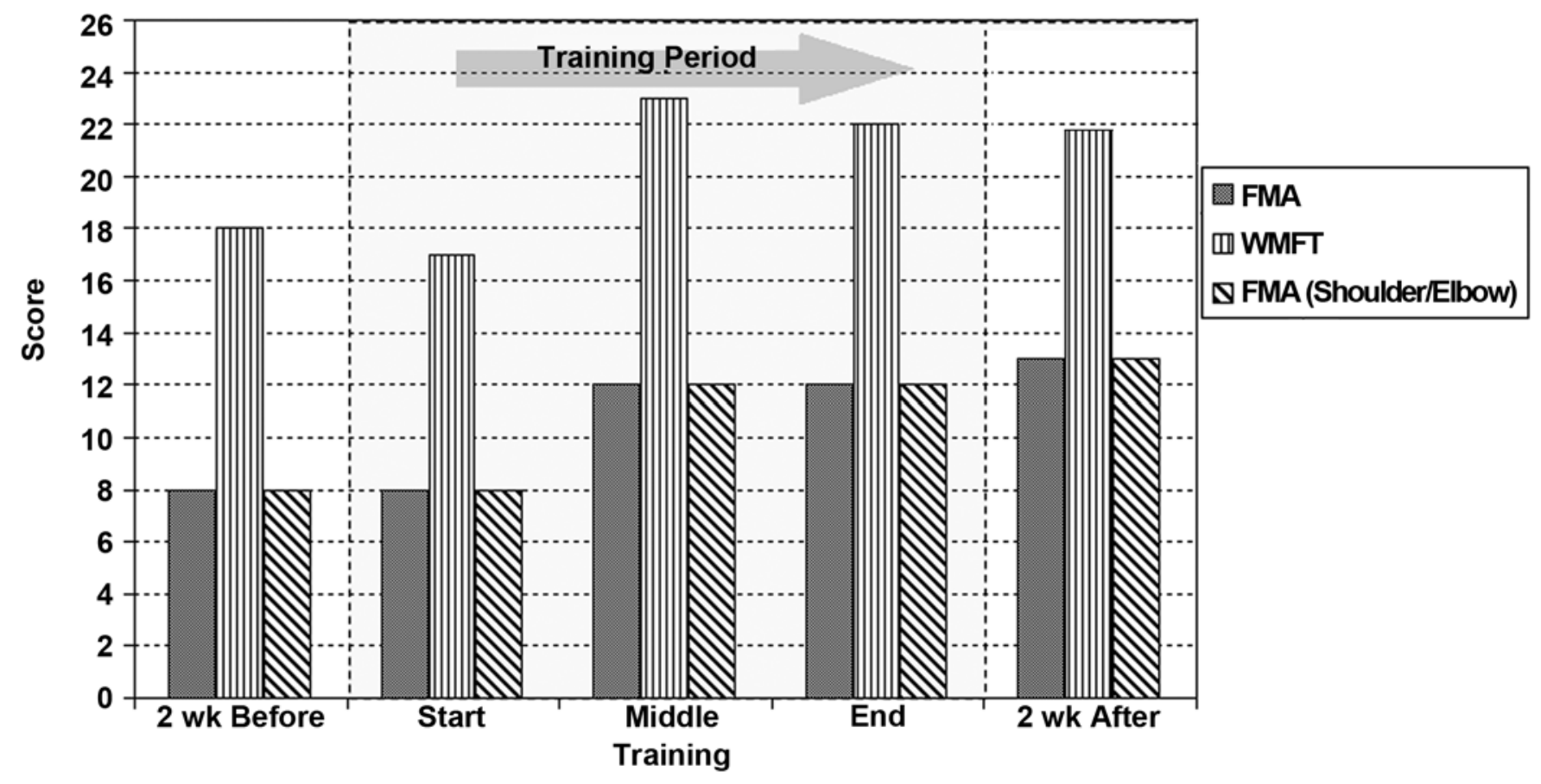

Figure 10.

Fugl-Meyer Motor Assessment (FMA) and Wolf Motor Function Test (WMFT) scores for subject B: Arm- and Reach-mode training.

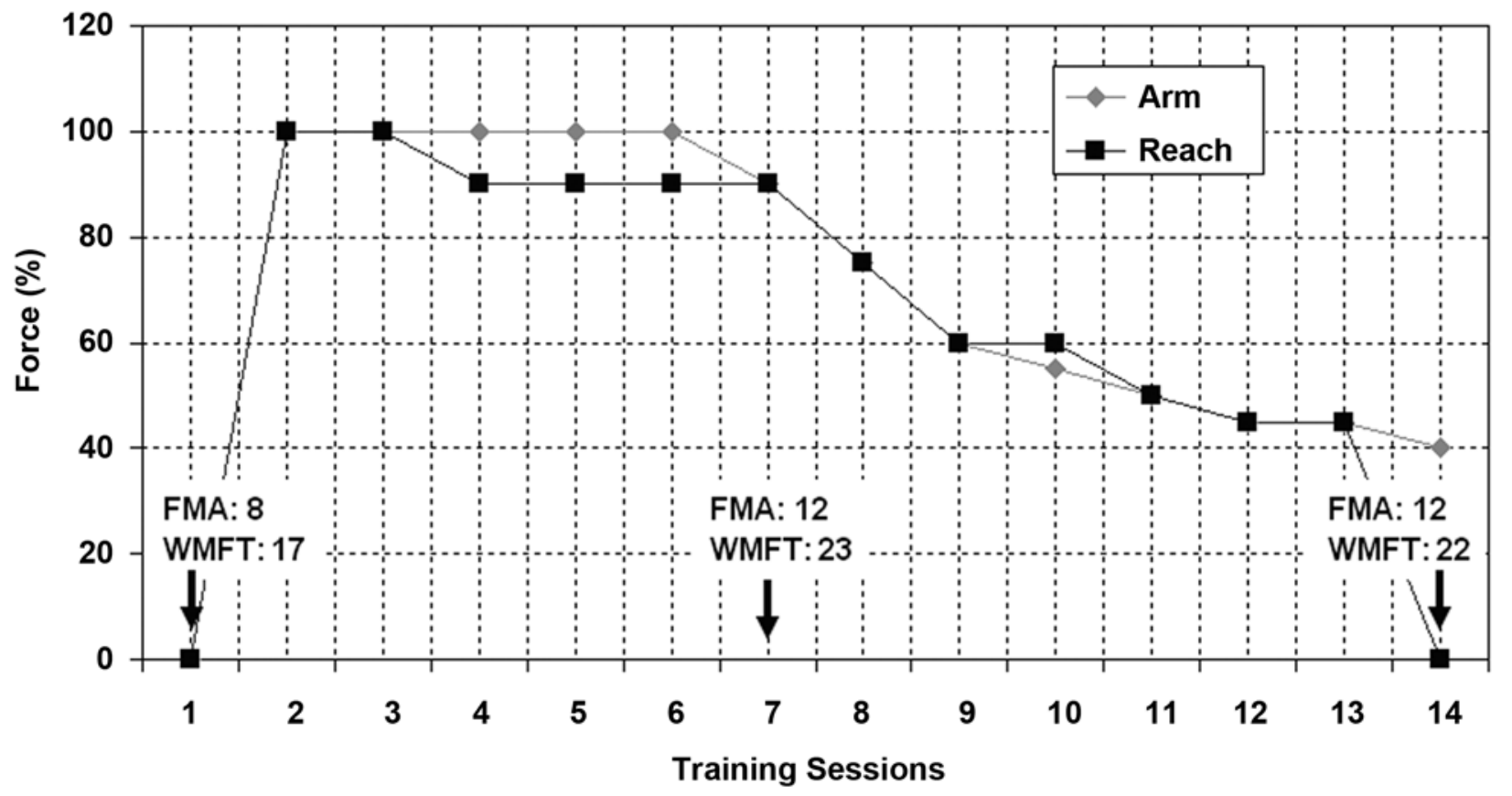

Figure 11.

Progression of reduced assistive forces by Universal Haptic Pantograph (UHP) (subject B) in Arm-mode training (Arm) and in Reach-mode training (Reach). Motion assistance provided by UHP is automatically reduced each session based on needs of subject. Fugl-Meyer Motor Assessment (FMA) and Wolf Motor Function Test (WMFT) scores measured during sessions 1, 7, and 14 are shown. 


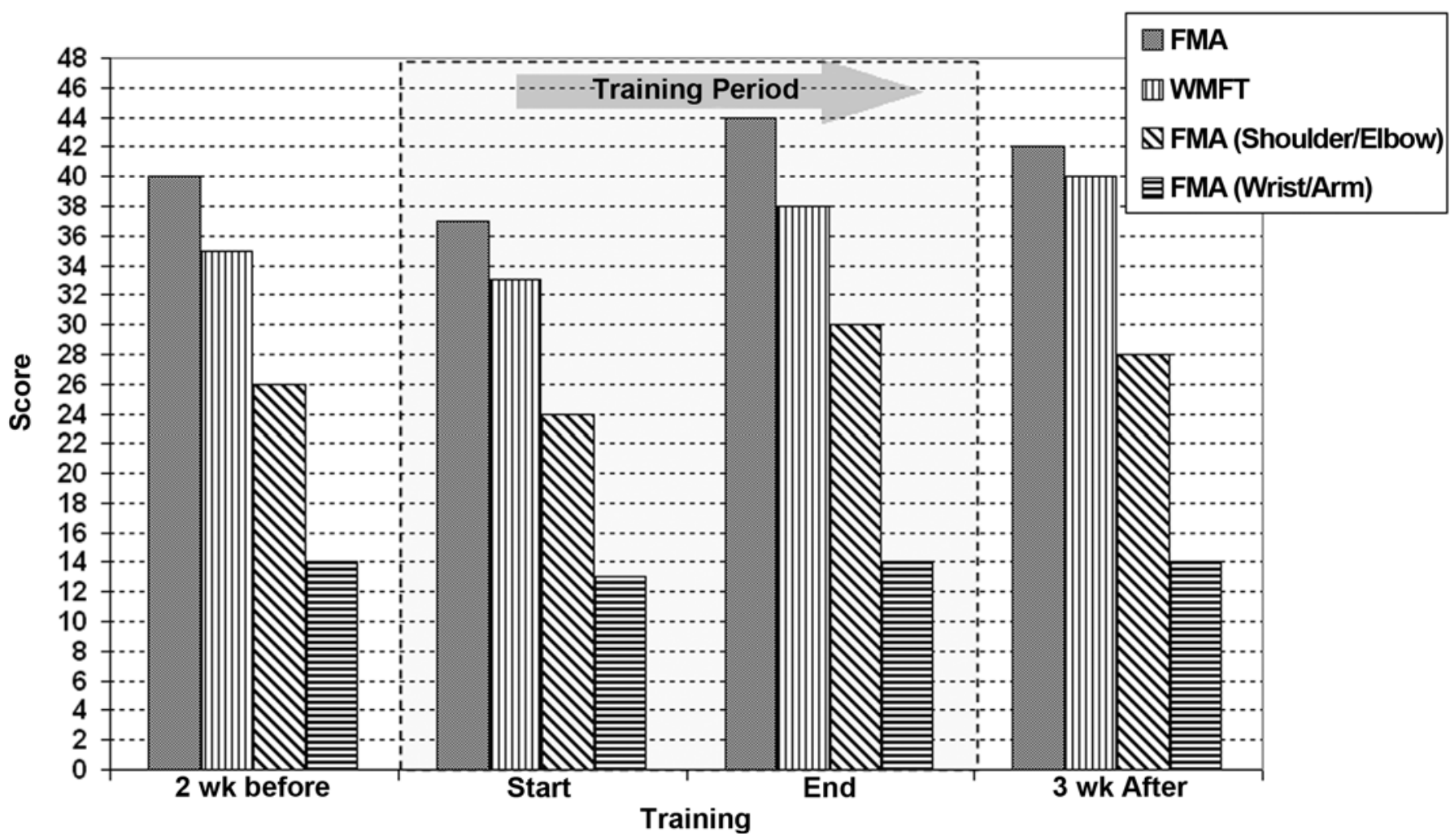

Figure 12.

Fugl-Meyer Motor Assessment (FMA) and Wolf Motor Function Test (WMFT) scores for subject C: Arm- and Reach-mode training.

achieves comparable performance to that achieved previously by precision motors. Therefore, with appropriately selected design parameters, the developed multifunction haptic device can significantly reduce the cost of rehabilitation robotic hardware while providing comparable performance to single-mode haptic devices.

The results of clinical testing with three subjects showed that movement of the shoulder, elbow, and wrist sustained up to 4 weeks posttreatment improved substantially. So far, we performed testing using the Wrist, Arm, and Reach modes, while additional clinical testing and interview with therapist could provide more objective and scientific evaluation of additional modes and usability of the developed mechanism.

\section{ACKNOWLEDGMENTS}

\section{Author Contributions:}

Study concept and design: Z. Matjačić, T. Keller, J. C. Perry, J. Oblak, J. H. Jung, I. Cikajlo, N. Goljar, N. Bizovičar, J. F. Veneman.

Acquisition of data: J. Oblak, I. Cikajlo, N. Goljar, N. Bizovičar, Z. Matjačić.
Analysis and interpretation of data: J. Oblak, Z. Matjačić. Drafting of manuscript: J. C. Perry, J. Oblak, J. H. Jung.

Critical revision of manuscript for important intellectual content:

J. F. Veneman.

Obtained funding: T. Keller, Z. Matjačić.

Administrative, technical, or material support: T. Keller, Z. Matjačić, J. H. Jung.

Study supervision: T. Keller, Z. Matjačić.

Financial Disclosures: The authors have declared that no competing interests exist.

Funding/Support: This material was based on work supported in part by FIK and the Spanish Ministry of Science and Innovation, project PID-020100-2009-21.

Institutional Review: The Slovenian National Medical Ethics Committee approved the experimental tests. All subjects gave informed consent. Participant Follow-Up: The authors do not plan to inform participants of the publication of this study. However, all participants were informed on entering the study that the results would be published in a scientific article.

\section{REFERENCES}

1. Harwin WS, Patton JL, Edgerton VR. Challenges and opportunities for robot-mediated neurorehabilitation. IEEE Spec Iss Med Robot. 2006;94(9):1717-26. 
2. Krebs HI, Palazzolo JJ, Dipietro L, Ferraro M, Krol J, Rannekleiv K, Volpe BT, Hogan N. Rehabilitation robotics: Performance-based progressive robot-assisted therapy. Auton Robots. 2003;15(1):7-20. DOI:10.1023/A:1024494031121

3. Hidler JM, Nichols D, Pelliccio M, Brady K. Advances in the understanding and treatment of stroke impairment using robotic devices. Top Stroke Rehabil. 2005;12(2):22-35. [PMID: 15940582] DOI:10.1310/RYT5-62N4-CTVX-8JTE

4. Krebs HI, Hogan N, Volpe BT, Aisen ML, Edelstein L, Diels C. Overview of clinical trials with MIT-MANUS: A robot-aided neuro-rehabilitation facility. Technol Health Care. 1999;7(6):419-23. [PMID: 10665675]

5. Fanin C, Gallina P, Rossi A, Zanatta U, Masiero S. NeReBot: A wire-based robot for neurorehabilitation. Proceedings of the 8th IEEE International Conference on Rehabilitation Robotics; 2003 Apr 22-25; Daejeon, Korea. Daejeon (Korea): Human-Friendly Welfare Robot System Engineering Research Center; 2003. p. 23-27.

6. Coote S, Stokes E, Murphy B, Harwin W. The effect of GENTLE/s robot-mediated therapy on upper extremity dysfunction post stroke. Proceedings of the 8th IEEE International Conference on Rehabilitation Robotics; 2003 Apr 22-25; Daejeon, Korea. Daejeon (Korea): Human-Friendly Welfare Robot System Engineering Research Center; 2003. p. 59-61.

7. Kahn LE, Lum PS, Rymer WZ, Reinkensmeyer DJ. Robotassisted movement training for the stroke-impaired arm: Does it matter what the robot does? J Rehabil Res Dev. 2006;43(5):619-30. [PMID: 17123203] DOI:10.1682/JRRD.2005.03.0056

8. Kayyali R, Shirmohammadi S, El Saddik A, Lemaire E. Daily-life exercises for haptic motor rehabilitation. Proceedings of IEEE International Workshop on Haptic Audio Visual Environments and Games; 2007 Oct 12-14; Ottawa, Canada. Los Alamitos (CA): IEEE Press; 2007. p. 118-23. DOI:10.1109/HAVE.2007.4371599

9. Van der Linde RQ, Lammertse P. HapticMaster-A generic force controlled robot for human interaction. Ind Robot. 2003; 30(6):515-24. DOI:10.1108/01439910310506783

10. ReoTherapy [Internet]. Trussville (AL): Motorika Medical Inc; 2008 [cited 2010 Mar 31]. Available from: http://www.motorika.com/.

11. Burgar CG, Lum PS, Shor PC, Machiel Van der Loos HF. Development of robots for rehabilitation therapy: The Palo Alto VA/Stanford experience. J Rehabil Res Dev. 2000:37(6); 663-73. [PMID: 11321002]

12. Spencer SJ, Klein J, Minakata K, Le V, Borrow JE, Reinkensmeyer DJ. A low cost parallel robot and trajectory optimization method for wrist and forearm rehabilitation using Wii. Proceedings of the 2nd IEEE RAS \& EMBS International Conference on Biomedical Robotics and Bio- mechatronics; 2008 Oct 19-22; Scottsdale, AZ. Los Alamitos (CA): IEEE Press; 2008. p. 869-74.

13. Gupta A, O'Malley MK. Design of a haptic arm exoskeleton for training and rehabilitation. IEEE AMSE Trans Mechatron. 2006;11(3):280-89.

14. Koeneman EJ, Schultz RS, Wolf SL, Herring DE, Koeneman JB. A pneumatic muscle hand therapy device. Conf Proc IEEE Eng Med Biol Soc. 2004;4:2711-13. [PMID: 17270836]

15. Takahashi CD, Der-Yeghiaian L, Le VH, Cramer SC. A robotic device for hand motor therapy after stroke. Proceedings of the 9th International Conference on Rehabilitation Robotics; 2005 Jun 28-Jul 1; Chicago, IL. Los Alamitos (CA): IEEE Press; 2005. p. 17-20.

DOI:10.1109/ICORR.2005.1501041

16. Hesse S, Schulte-Tigges G, Konrad M, Bardeleben A, Werner C. Robot-assisted arm trainer for the passive and active practice of bilateral forearm and wrist movements in hemiparetic subjects. Arch Phys Med Rehabil. 2003;84(6): 915-20. [PMID: 12808550]

DOI:10.1016/S0003-9993(02)04954-7

17. Sanchez RJ Jr, Wolbrecht E, Smith R, Liu J, Cramer S, Rahman T, Bobrow JE, Reinkensmeyer DJ. A pneumatic robot for re-training arm movement after stroke: Rationale and mechanical design. Proceedings of the 9th International Conference on Rehabilitation Robotics; 2005 Jun 28-Jul 1; Chicago, IL. Los Alamitos (CA): IEEE Press; 2005. p. 500-504. DOI:10.1109/ICORR.2005.1501151

18. Nef T, Mihelj M, Kiefer G, Perndl C, Müller R, Riener R. ARMin-Exoskeleton for arm therapy in stroke patients. Proceedings of the 10th International Conference on Rehabilitation Robotics; 2007 Jun 13-15; Noordwijk, the Netherlands. Los Alamitos (CA): IEEE Press; 2007. p. 68-74.

DOI:10.1109/ICORR.2007.4428408

19. Stienen AHA, Hekman EEG, Van der Helm FCT, Prange GB, Jannink MJA, Aalsma AMM, Van der Kooij H. Dampace: Dynamic force-coordination trainer for the upper extremities. Proceedings of the 10th International Conference on Rehabilitation Robotics; 2007 Jun 13-15; Noordwijk, the Netherlands. Los Alamitos (CA): IEEE Press; 2007. p. 820-26. DOI:10.1109/ICORR.2007.4428519

20. Sanchez RJ, Liu J, Rao S, Shah P, Smith R, Rahman T, Cramer SC, Bobrow JE, Reinkensmeyer DJ. Automating arm movement training following severe stroke: Functional exercises with quantitative feedback in a gravity-reduced environment. IEEE Trans Neural Syst Rehabil Eng. 2006; 14(3):378-89. [PMID: 17009498] DOI:10.1109/TNSRE.2006.881553

21. Krebs HI, Volpe BT, Williams D, Celestino J, Charles SK, Lynch D, Hogan N. Robot-aided neurorehabilitation: A robot for wrist rehabilitation. IEEE Trans Neural Syst 
Rehabil Eng. 2007;15(3):327-35. [PMID: 17894265] DOI:10.1109/TNSRE.2007.903899

22. Toth A, Fazekas G, Arz G, Jurak M, Horvath M. Passive robotic movement therapy of the spastic hemiparetic arm with REHAROB: Report of the first clinical test and the follow-up system improvement. Proceedings of the 9th International Conference on Rehabilitation Robotics; 2005 Jun 28-Jul 1; Chicago, IL. Los Alamitos (CA): IEEE Press; 2005. p. 127-30. DOI:10.1109/ICORR.2005.1501067

23. Perry JC, Powell JM, Rosen J. Isotropy of an upper limb exoskeleton and the kinematics and dynamics of the human arm. Appl Bionics Biomech. 2009;6(2):175-91.

DOI:10.1080/11762320902920575

24. Yeong CF, Melendez-Calderon A, Burdet E. Analysis of pick-and-place, eating and drinking movements for the workspace definition of simple robotic devices. Proceedings of the IEEE International Conference on Rehabilitation Robotics; 2009 Jun 23-26; Kyoto, Japan. Los Alamitos (CA): IEEE Press; 2009. p. 46-52. DOI:10.1109/ICORR.2009.5209475

25. Sukal TM, Ellis MD, Dewald JP. Shoulder abductioninduced reductions in reaching work area following hemiparetic stroke: Neuroscientific implications. Exp Brain Res. 2007;183(2):215-23. [PMID: 17634933] DOI:10.1007/s00221-007-1029-6

26. Ellis MD, Sukal-Moulton T, Dewald JPA. Progressive shoulder abduction loading is a crucial element of arm rehabilitation in chronic stroke. Neurorehabil Neural Repair. 2009;23(8):862-69. [PMID: 19454622]

DOI: $10.1177 / 1545968309332927$

27. Oblak J, Cikajlo I, Matjačić Z. Universal haptic drive: A robot for arm and wrist rehabilitation. IEEE Trans Neural Syst Rehabil Eng. 2010;18(3):293-302. [PMID: 19846386] DOI:10.1109/TNSRE.2009.2034162

28. Craig JJ. Introduction to robotics. Mechanics and control. 2nd ed. Reading (MA): Addison-Wesley; 1989. p. 277-80.

Submitted for publication March 31, 2010. Accepted in revised form October 6, 2010.

This article and any supplementary material should be cited as follows:

Perry JC, Oblak J, Jung JH, Cikajlo I, Veneman JF, Goljar N, Bizovičar N, Matjačić Z, Keller T. Variable structure pantograph mechanism with spring suspension system for comprehensive upper-limb haptic movement training. J Rehabil Res Dev. 2011;48(4):317-34.

DOI:10.1682/JRRD.2010.03.0043

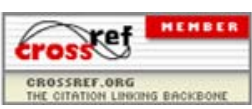


